\title{
Theoretical model for the structural phase transition at the metal-insulator transition in polymerized $\mathrm{KC}_{60}$
}

\author{
B. Verberck, ${ }^{1}$ A. V. Nikolaev, ${ }^{1,2}$ and K. H. Michel ${ }^{1}$ \\ ${ }^{1}$ Department of Physics, University of Antwerp, Universitaire Instelling Antwerpen, Universiteitsplein 1, 2610 Antwerp, Belgium \\ ${ }^{2}$ Institute of Physical Chemistry of the Russian Academy of Sciences, Leninskii Prospect 31, 117915, Moscow, Russia
}

(Received 2 May 2002; revised manuscript received 16 July 2002; published 29 October 2002)

\begin{abstract}
The recently discovered structural transition in polymerized $\mathrm{KC}_{60}$ at about $50 \mathrm{~K}$ results in a doubling of the unit cell volume and accompanies the metal-insulator transition. Here we show the $(\vec{a}+\vec{c}, \vec{b}, \vec{a}-\vec{c})$ superstructure results from small orientational charge density waves along the polymer chains and concomitant displacements of the surrounding $\mathrm{K}^{+}$ions. The effect is specific for the space group Pmnn of $\mathrm{KC}_{60}$ and is absent in $\mathrm{RbC}_{60}$ and $\mathrm{CsC}_{60}$ (space group $I 2 / m$ ). The mechanism is relevant for the metal-insulator transition.

DOI: 10.1103/PhysRevB.66.165425

PACS number(s): 64.60.-i, 64.70.-p, 72.10.-d, 72.15.-v
\end{abstract}

\section{INTRODUCTION}

Among the alkali-metal-doped fullerides $A_{x} \mathrm{C}_{60}$, where $A=\mathrm{K}, \mathrm{Rb}, \mathrm{Cs}$, the $x=1$ compounds ${ }^{1}$ have attracted attention, because they form plastic cubic crystalline phases with rocksalt structure at high temperature $(T>350 \mathrm{~K})$ and stable polymeric phases $^{2}$ of reduced symmetry at lower $T$. In addition, via rapid quenching, a metastable dimer phase was obtained. ${ }^{3,4}$ It was suggested that the dimer structure should be a Peierls insulator. ${ }^{3}$ In the following we will restrict ourselves to the polymer phases. There, the $\mathrm{C}_{60}$ molecules are linked through a $[2+2]$ cycloaddition and form chains along the former cubic [110] direction. The orientation of the polymer chains is characterized by the angle $\psi$ of the planes of cycloaddition with the former cubic [001] direction. The structure of $\mathrm{KC}_{60}$ is orthorhombic ${ }^{5,6}$ [space group Pmnn; Fig. 1(a)]; the orthorhombic $\vec{a}$ axis is parallel to the axis of polymerization and the $\vec{c}$ axis parallel to the former cubic [001] direction. The Pmnn structure is characterized by alternating orientations $\pm \psi$ of the polymer chains in successive $(\vec{a}, \vec{b})$ planes and the same orientation of the chains within one plane, $|\psi| \approx 45^{\circ}$. On the other hand, the structure of both polymerized $\mathrm{RbC}_{60}$ (Ref. 6) and $\mathrm{CsC}_{60}$ (Ref. 7) is monoclinic, space group $I 2 / m$ [Fig. 1(b)]. Here the polymer chains have the same orientation $\psi$ not only within the same $(\vec{a}, \vec{b})$ plane but also in successive (001) planes.

In addition to the structural differences, the electronic and magnetic properties of $\mathrm{KC}_{60}$ on one hand and $\mathrm{RbC}_{60}, \mathrm{CsC}_{60}$, on the other hand, are different (for a review, see Ref. 8). Neither the differences in structure nor those in electromagnetic properties can be simply related to the relatively small differences in the lattice constants among the $A \mathrm{C}_{60}$ compounds. We then conclude that more alkali-metal-specific effects are relevant. To explain the structural differences of the polymer phases of $\mathrm{KC}_{60}$ (space group Pmnn) and $\mathrm{RbC}_{60}$ and $\mathrm{CsC}_{60}$ (space group $I 2 / \mathrm{m}$ ), it has turned out that the quadrupolar polarizability of the alkali-metal ions is the decisive alkali-metal-specific characteristic. ${ }^{9,10}$ The quadrupolar polarizability of the alkali-metal ion $A^{+}$is related to the average radius $d_{A}$ of its first valence electron $d$ shell. Indeed, $d_{\mathrm{Rb}}=1.82 \AA$ and $d_{\mathrm{Cs}}=1.87 \AA$ are close to each other but quite different from $d_{\mathrm{K}}=1.47 \AA$. The partial occupancy of the first excited $d$ state in the solids is possible because of the large interstitial space available for the alkali-metals, this relatively large space being a unique property of the $A C_{60}$ compounds.

Recently, a combined study of the electronic and structural properties of $\mathrm{KC}_{60}$ has been carried out by Coulon et al.: ${ }^{11} \mathrm{x}$-ray diffraction studies have revealed a structural phase transition in polymerized $\mathrm{KC}_{60}$ at $60 \mathrm{~K} \leqslant T_{c} \leqslant 65 \mathrm{~K}$, while ESR measurements have shown the existence of a metal-insulator transition at $T \approx 50 \mathrm{~K}$, which stresses once again the close connection between structural and electronic properties in the $A \mathrm{C}_{60}$ alkali-metal fullerides. Above the structural critical temperature $T_{c}$, the Bravais lattice of polymerized $\mathrm{KC}_{60}$ is orthorhombic; at $T_{c}$, the crystal changes to a $(\vec{a}+\vec{c}, \vec{b}, \vec{a}-\vec{c})$ superstructure, which can be viewed as a doubling of the basis vectors $\vec{a}$ and $\vec{c}$. Concerning the possible nature of this structural phase transition (i.e., the doubling mechanism), it was pointed out by Coulon et al. ${ }^{11}$ (i) that displacements of the $\mathrm{C}_{60}$ centers of mass can be ruled out and (ii) that a combination of a charge modulation on the $\mathrm{C}_{60}$ monomers and large correlated $\mathrm{K}$ displacements is an appealing hypothesis. a

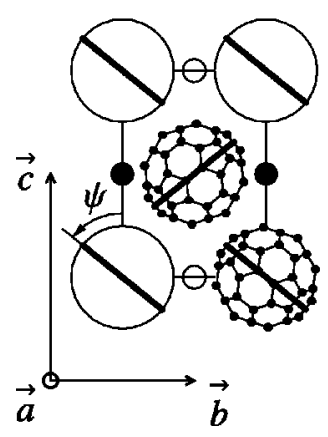

b

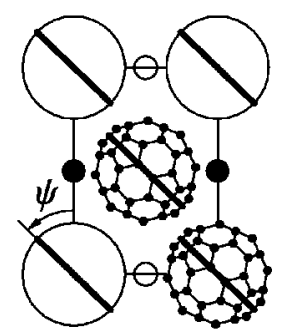

FIG. 1. Crystal structures projected onto the crystallographic $(\vec{b}, \vec{c})$ plane: (a) Pmnn, (b) $I 2 / m$. The thick bars represent the projection of the cycloaddition planes. Polymerization occurs along $\vec{a}$. The alkali-metals located in $(\vec{b}, \vec{c})$ planes and at $\pm \vec{a} / 2$ are denoted by full and empty circles. 
We note that such a combined structural and metalinsulator phase transition has not yet been observed in $\mathrm{RbC}_{60} \cdot{ }^{12}$ Bearing in mind the structural differences of the polymer phases of $\mathrm{KC}_{60}$ and $\mathrm{RbC}_{60}$, the presence/absence of a second structural phase transition in $A \mathrm{C}_{60}$ seems to be related to the precise orientations of the polymer chains in $P m n n$ and $I 2 / m$, respectively.

Here we propose a doubling mechanism that accounts for the observed structural phase transition in $\mathrm{KC}_{60}$ on one hand, and for the absence of such a transition in $\mathrm{RbC}_{60}$ on the other hand, both in agreement with the present experimental knowledge of these compounds.

\section{DOUBLING MECHANISM}

We consider rigid $\mathrm{KC}_{60}$ and $\mathrm{RbC}_{60}$ crystals in the polymerized phase. For $\mathrm{KC}_{60}$, this phase has space group Pmnn, while for $\mathrm{RbC}_{60}$ the space group is $I 2 / \mathrm{m}$. The $I 2 / \mathrm{m}$ space group has a monoclinic Bravais lattice; we will treat it as an orthorhombic lattice, however, since the "monoclinic" angle $\alpha=90.316^{\circ}$ of $\mathrm{RbC}_{60}$ obtained by neutron scattering measurements ${ }^{13}$ is extremely close to $90^{\circ}$. The centers of mass of the $N \mathrm{C}_{60}$ monomers are then located on the lattice points of a body-centered orthorhombic lattice with basis vectors $\vec{a}=a \vec{e}_{X}, \vec{b}=b \vec{e}_{Y}$, and $\vec{c}=c \vec{e}_{Z}$, where $\left(\vec{e}_{X}, \vec{e}_{Y}, \vec{e}_{Z}\right)$ are the basis vectors of the underlying Cartesian coordinate system. The axes of polymerization are parallel to $\vec{a}$. A lattice point is labeled by indices $\vec{n}=\left(n_{1}, n_{2}, n_{3}\right)$, which are either all integer numbers or all integer numbers $+\frac{1}{2}$, corresponding, respectively, to the corner and the center points of the unit cells. The position vector of lattice point $\vec{n}$ reads

$$
\vec{X}(\vec{n})=n_{1} \vec{a}+n_{2} \vec{b}+n_{3} \vec{c} .
$$

To each polymer chain, a rotation angle $\psi$ can be assigned. We let $\psi=0$ correspond to the situation where the polymer chain is in the standard orientation, which is defined as the orientation where the plane of cycloaddition is parallel to the $(\vec{a}, \vec{c})$ plane. The angle $\psi$ then measures a counterclockwise rotation of the polymer chain about $\vec{a}$. More generally, the orientation angle can be seen as a property of a single $\mathrm{C}_{60}$ monomer; therefore we write $\psi \equiv \psi(\vec{n})$. However, since all the $\mathrm{C}_{60}$ monomers in the same polymer chain have the same orientation angle and since a polymer chain can be addressed by the indices $\left(n_{2}, n_{3}\right), \psi$ is independent of the index $n_{1}$. Furthermore, the Pmnn structure is characterized by an alternation of the orientations of the polymer chains along the $\vec{c}$ axis only; hence for $\mathrm{KC}_{60} \psi$ is also independent of the index $n_{2}$ :

$$
\psi(\vec{n}) \equiv \psi\left(n_{3}\right)=(-1)^{2 n_{3}} \psi_{\mathrm{KC}_{60}} .
$$

The structure $I 2 / \mathrm{m}$ is characterized by an equal orientation of all the polymer chains in the crystal. Therefore, one has for $\mathrm{RbC}_{60}$ :

$$
\psi(\vec{n}) \equiv \psi_{\mathrm{RbC}_{60}} .
$$

The chain orientation angles have been redetermined recently by neutron scattering experiments: ${ }^{13} \quad \psi_{\mathrm{KC}_{60}}=50^{\circ}$, $\psi_{\mathrm{RbC}_{60}}=46^{\circ}$.

Due to the charge transfer of one electron from an alkalimetal atom to a $\mathrm{C}_{60}$ molecule, a charge distribution $\rho$ with total charge $-e$ exists on every $\mathrm{C}_{60}$ monomer. For a given $\mathrm{C}_{60}$ monomer $\vec{n}$, we introduce a Cartesian coordinate system with basis vectors $\left(\vec{e}_{x}, \vec{e}_{y}, \vec{e}_{z}\right)$ and the center of mass of the monomer as origin. The charge distribution can then be written as

$$
\rho \equiv \rho(\vec{r} ; \psi(\vec{n}))=\rho(x, y, z ; \psi(\vec{n})),
$$

where $\vec{r}=x \vec{e}_{x}+y \vec{e}_{y}+z \vec{e}_{z}$

As a mechanism for the doubling of the lattice basis vectors $\vec{a}$ and $\vec{c}$, we suggest the following: while retaining the rigid structure for the $C$ nuclei and the closed $\pi$ - and $\sigma$-shell electrons, small orientational deviations of the valence electron density $\rho$ on every $\mathrm{C}_{60}$ monomer from this structure are allowed to occur, in such a way that these deviations (and therefore equivalent lattice points) have periodicities $2 \vec{a}, \vec{b}$, and $2 \vec{c}$. A rotation of the electron density can occur because there are orbital degrees of freedom for one valence electron on the threefold degenerate $t_{1 u}$ lowest unoccupied molecular orbital (LUMO) level. ${ }^{14}$ The valence electron density depends on the coefficients of expansion in terms of these three orbitals and this leads to its effective rotation. We will limit ourselves to rotations of the charge distributions about the polymer chain axes. To denote the angular deviation of the charge distribution from $\psi(\vec{n})$, we use the notation $\Delta \psi(\vec{n})$ :

$$
\rho \equiv \rho(\vec{r} ; \psi(\vec{n})+\Delta \psi(\vec{n}))=\rho(x, y, z ; \psi(\vec{n})+\Delta \psi(\vec{n})) .
$$

In order to have the desired periodicity changes, we take

$$
\begin{aligned}
\Delta \psi\left(n_{1}, n_{2}, n_{3}\right) & =\Delta \psi\left(n_{1}+\frac{1}{2}, n_{2}+\frac{1}{2}, n_{3}+\frac{1}{2}\right) \\
& =\left\{\begin{array}{l}
+\Delta \psi_{0} \text { if } n_{1}+n_{3} \text { even }, \\
-\Delta \psi_{0} \text { if } n_{1}+n_{3} \text { odd },
\end{array}\right.
\end{aligned}
$$

with $n_{1}, n_{2}, n_{3} \in \mathbb{Z}$. In Eq. (2.6), $\Delta \psi_{0}$ represents the angle measuring the deviation from $\psi$. The mechanism is illustrated schematically in Fig. 2. In Sec. IV we will comment on the charge distribution model to be used.

It is convenient to introduce spherical coordinates $(r, \theta, \phi)$, related to the Cartesian coordinates $(x, y, z)$ via

$$
\begin{gathered}
y=r \sin \theta \cos \phi, \\
z=r \sin \theta \sin \phi, \\
x=r \cos \theta,
\end{gathered}
$$

since a counterclockwise rotation of the charge distribution $\rho$ over an angle $\psi+\Delta \psi$ about the $\vec{a}$ axis is then simply achieved by replacing $\phi$ by $\phi-\psi-\Delta \psi$ : 


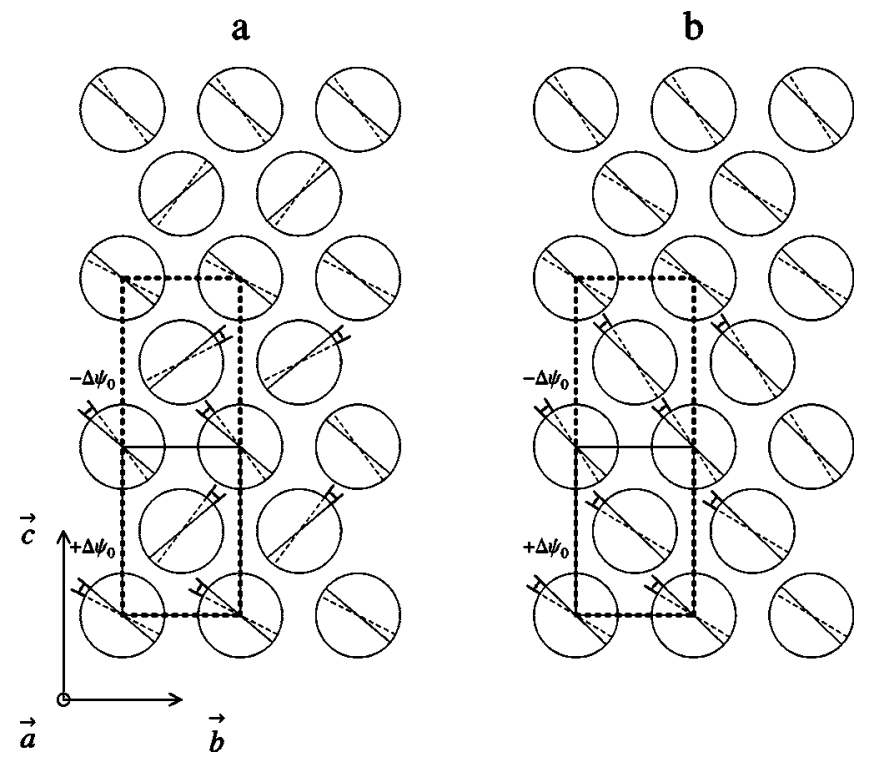

FIG. 2. Illustration of the proposed doubling mechanism. Shown is a projection of the $\mathrm{C}_{60}$ molecules, symbolized by circles, onto the $(\vec{b}, \vec{c})$ crystallographic plane (compare with Fig. 1). The alkalimetal atoms have been omitted for clarity. The solid and dashed lines in the circles represent the orientations of the charge distributions: (a) $\mathrm{KC}_{60}$, (b) $\mathrm{RbC}_{60}$. The solid lines correspond to the original (a) Pmnn and (b) $I 2 / m$ structures, the suggested doubling mechanism consists of angular deviations of the electronic density distributions as indicated by the dashed lines. The proposed scheme results in a doubling of the lattice vector $\vec{c}$, while the lattice vector $\vec{b}$ remains the same. The original (projection of the) unit cell is shown as a solid frame, the (projection of the) unit cell taking into account the electronic deviations is shown as a dashed frame. Along the $\vec{a}$ axis, a doubling pattern (angular deviations of the charge distributions) similar to the doubling along the $\vec{c}$ axis occurs.

$$
\rho(r, \theta, \phi ; \psi(\vec{n})+\Delta \psi(\vec{n}))=\rho(r, \theta, \phi-\psi(\vec{n})-\Delta \psi(\vec{n}) ; 0) .
$$

We simplify the notation and write from now on:

$$
\rho(r, \theta, \phi) \equiv \rho(r, \theta, \phi ; 0) .
$$

\section{POTENTIAL ENERGY}

In order to determine whether the above-described electronic density distortions can occur or not, it is necessary to investigate the potential energy of the crystal. We consider the contribution $\mathcal{U}$ to the potential energy arising from $\mathrm{C}_{60}-\mathrm{C}_{60}$ interactions, which can be written as a sum of pair potentials:

$$
\mathcal{U}=\frac{1}{2} \sum_{\vec{n}, \vec{n}^{\prime}} U\left(\vec{n}, \vec{n}^{\prime}\right)
$$

Since we are interested in how the potential energy of the crystal is influenced by the above-described orientational deviations $\Delta \psi(\vec{n})$ of the charge distributions on all $\mathrm{C}_{60}{ }^{-}$monomers, only electronic interactions have to be taken into account (van der Waals-type interactions refer to the neutral $\mathrm{C}$ cores, which in our model are assumed to constitute a rigid structure). The electrostatic energy of two $\mathrm{C}_{60}{ }^{-}$monomers at lattice points $\vec{n}$ and $\vec{n}^{\prime}$ is given by

$$
U\left(\vec{n}, \vec{n}^{\prime}\right)=\frac{1}{4 \pi \epsilon_{0}} \int d \vec{r} \int d \overrightarrow{r^{\prime}} \frac{\rho(\vec{r} ; \alpha(\vec{n})) \rho\left(\vec{r}^{\prime} ; \alpha\left(\vec{n}^{\prime}\right)\right)}{\left|\vec{r}-\vec{r}^{\prime}-\vec{X}\left(\vec{n}^{\prime}-\vec{n}\right)\right|}
$$

where we have introduced the notation

$$
\alpha(\vec{n}) \equiv \psi(\vec{n})+\Delta \psi(\vec{n}) .
$$

The integration variables $\vec{r}$ and $\vec{r}^{\prime}$ in Eq. (3.2a) refer to the local coordinate systems associated with the respective lattice sites $\vec{n}$ and $\vec{n}^{\prime}$; hence the appearance of the relative position vector $\vec{X}\left(\vec{n}^{\prime}-\vec{n}\right)=\vec{X}\left(\vec{n}^{\prime}\right)-\vec{X}(\vec{n})$. Using the previously introduced spherical coordinates, $U\left(\vec{n}, \vec{n}^{\prime}\right)$ can be rewritten as

$$
\begin{aligned}
& U\left(\vec{n}, \vec{n}^{\prime}\right)= \frac{1}{4 \pi \epsilon_{0}} \int_{0}^{\infty} r^{2} d r \int_{0}^{\pi} \sin \theta d \theta \int_{0}^{2 \pi} d \phi \int_{0}^{\infty} r^{\prime 2} d r^{\prime} \int_{0}^{\pi} \sin \theta^{\prime} d \theta^{\prime} \\
& \times \int_{0}^{2 \pi} d \phi^{\prime} \frac{\rho(r, \theta, \phi-\psi(\vec{n})-\Delta \psi(\vec{n})) \rho\left(r^{\prime}, \theta^{\prime}, \phi^{\prime}-\psi\left(\vec{n}^{\prime}\right)-\Delta \psi\left(\vec{n}^{\prime}\right)\right)}{\left|\vec{r}-\vec{r}^{\prime}-\vec{X}\left(\vec{n}^{\prime}-\vec{n}\right)\right|} \\
&\left|\vec{r}-\vec{r}^{\prime}-\vec{X}\left(\vec{n}^{\prime}-\vec{n}\right)\right|=\left\{\left[r \cos \theta-r^{\prime} \cos \theta^{\prime}-\left(n_{1}^{\prime}-n_{1}\right) a\right]^{2}+\left[r \sin \theta \cos \phi-r^{\prime} \sin \theta^{\prime} \cos \phi^{\prime}-\left(n_{2}^{\prime}-n_{2}\right) b\right]^{2}\right. \\
&\left.+\left[r \sin \theta \sin \phi-r^{\prime} \sin \theta^{\prime} \sin \phi^{\prime}-\left(n_{3}^{\prime}-n_{3}\right) c\right]^{2}\right\}^{1 / 2} .
\end{aligned}
$$

As will be discussed in more detail in Sec. IV, the charge distribution $\rho$ can be expanded in even multipoles $l$ $=0,2,4, \ldots$. The lowest-order term containing angular dependence will therefore be a monopole-quadrupole interaction. As a consequence, the angular dependent part of the potential energy $U$, being of the Coulomb-type, decreases fast enough with the distance, and it is justified to consider a limited number of nearest-neighbor interactions. We write Eq. (3.1) as 


$$
\mathcal{U}=\frac{1}{2} \sum_{\vec{n}} V(\vec{n}),
$$

where

$$
V(\vec{n})=\sum_{\vec{\mu}} U(\vec{n}, \vec{n}+\vec{\mu})
$$

The summation over $\vec{\mu}$ runs over the fourteen nearestneighbor sites with relative indices $\vec{\mu}=( \pm 1,0,0), \quad(0$, $\pm 1,0)$, $(0,0, \pm 1)$, $\left( \pm \frac{1}{2}, \pm \frac{1}{2}, \pm \frac{1}{2}\right)$. An analysis of the occuring orientations of the charge distributions on the various $\mathrm{C}_{60}{ }^{-}$monomers reveals that only two different types of lattice sites exist: if we let $n_{1}, n_{2}$, and $n_{3}$ be integers, then all sites with indices $\left(n_{1}, n_{2}, n_{3}\right)$ or $\left(n_{1}+\frac{1}{2}, n_{2}+\frac{1}{2}, n_{3}+\frac{1}{2}\right)$, satisfying $n_{1}+n_{3}$ even, have an equivalent neighborhood. We call these sites type I sites. The other sites $\left(n_{1}+n_{3}\right.$ odd $)$, which we label as type II sites, have also an equivalent environment, but one that is different from the type I neighborhood. We summarize this observation by writing

$$
\begin{aligned}
V\left(n_{1}, n_{2}, n_{3}\right) & =V\left(n_{1}+\frac{1}{2}, n_{2}+\frac{1}{2}, n_{3}+\frac{1}{2}\right) \\
& = \begin{cases}V^{\mathrm{I}} & \text { if } n_{1}+n_{3} \text { even } \\
V^{\mathrm{II}} & \text { if } n_{1}+n_{3} \text { odd, }\end{cases}
\end{aligned}
$$

with $n_{1}, n_{2}, n_{3} \in \mathbb{Z}$. Note that Eq. (3.6) is consistent with the imposed periodicity conditions (2.6). The potential energy of the entire crystal due to all electrostatic $\mathrm{C}_{60}{ }^{-}-\mathrm{C}_{60}{ }^{-}$interactions is obtained by carrying out the summation of Eq. (3.4), which runs over all lattice points:

$$
\mathcal{U}=\frac{N}{4}\left(V^{\mathrm{I}}+V^{\mathrm{II}}\right) \text {. }
$$

The functions $V^{\mathrm{I}}$ and $V^{\mathrm{II}}$ depend only on the angle $\Delta \psi_{0}$, introduced in Eq. (2.6), which is a measure for the deviation from the undistorted structure. To emphasize this dependence, we write

$$
\mathcal{U} \equiv \mathcal{U}\left(\Delta \psi_{0}\right)
$$

\section{CHARGE DISTRIBUTION}

The key point in the proposed model is the expression for the electronic density $\rho(\vec{r})$ of a $\mathrm{C}_{60}{ }^{-}$unit in a polymer chain. Polymerization leads to a reduction of the symmetry (in comparison with the icosahedral symmetry of $\mathrm{C}_{60}$ ) and the charge distribution can be expanded in multipoles with $l$ $=0,2,4, \ldots$. In earlier theoretical work, ${ }^{9,10}$ a quadrupolar charge distribution model was used in explaining the structural phase transition from the cubic (unpolymerized) to the orthorhombic (polymerized) phase of the $A \mathrm{C}_{60}$ alkali-metal fullerides. By using a simplified Slater-Koster tight-binding approach to determine $\rho(\vec{r})$, it has been shown ${ }^{15}$ that such a quadrupolar model is a reasonable first approximation. (Due to the $D_{2 h}$ symmetry of a polymer chain, only even multipoles occur.) However, by experimenting with various qua- drupolarlike electronic densities, we find that the energy $\mathcal{U}$ depends very sensitively on the precise location of the point charges used in constructing the charge distribution and that here no conclusion can be drawn based upon quadrupolar models. In our view, it is necessary to go beyond the quadrupolar contribution and to take into account higher multipoles. Indeed, if one examines the expansion of the angular part $\rho_{a}(\theta, \phi)$ of the charge distribution in terms of spherical harmonics, calculated in Ref. 15, one sees the relevance of the higher-order terms. In particular, the $(l=8, m=8)$ term seems to play an important role. The importance of relatively-high-order multipoles is not surprising: for example, orientational ordering in solid $\mathrm{C}_{60}$ (fullerite) is described using molecular and site-symmetry-adapted functions (SAFs) belonging to the manifolds $l=6,10,12$. $^{16}$ Furthermore, in expanding the van der Waals interactions between polymers in $A \mathrm{C}_{60}$ in terms of SAFs, we have recently discovered a similar pattern: the term with $(l=8, m$ $=8$ ) is remarkably dominant.

These observations lead us to use the electronic density of Ref. 15, which can be written as

$$
\rho(r, \theta, \phi)=-e \rho_{r}(r) \rho_{a}(\theta, \phi),
$$

where the charge of the electron $-e$ has been factored out in order to ensure that the angular part $\rho_{a}$ is dimensionless. The radial part $\rho_{r}$ is not relevant for our purposes; we locate the charge on a sphere with radius $R=3.55 \AA$, which is the radius of a $\mathrm{C}_{60}$ molecule: ${ }^{17}$

$$
\rho_{r}(r)=\frac{\delta(r-R)}{R^{2}} .
$$

If a more refined model for $\rho_{r}$ is used, the main effect is a renormalization of the intersite interactions. On the other hand, the angular part $\rho_{a}$ is anisotropic and results in multipole interactions of different sign and magnitude. It is given by

$$
\begin{aligned}
\rho_{a}(\theta, \phi)= & {\left[-0.70699 Y_{5}^{1, s}(\theta, \phi)+0.30659 Y_{5}^{3, s}(\theta, \phi)\right.} \\
& \left.+0.63731 Y_{5}^{5, s}(\theta, \phi)\right]^{2} .
\end{aligned}
$$

In Eq. (4.1c), $\rho_{a}$ is written as the modulus squared of the wave function $\psi_{3}(\theta, \phi)$, which is one of the three degenerate $t_{1 u}$ functions of a $\mathrm{C}_{60}{ }^{-}$ion. ${ }^{18}$ The real functions $Y_{l}^{m, s}(\theta, \phi)$ are the sine spherical harmonics defined in Ref. 19. Note that Eq. (4.1c) is exact within the framework of the tight-binding approach. In Fig. 3, the angular electron density $\rho_{a}$ for a $\mathrm{C}_{60}{ }^{-}$ion in the standard orientation is shown, projected onto the $(\vec{b}, \vec{c})$ and $(\vec{a}, \vec{c})$ planes. The charge is mainly concentrated in the equatorial region $(x=0$ or, equivalently, $\theta$ $=\pi / 2$ ), in agreement with recent NMR results. ${ }^{20}$ As can be seen clearly in Fig. 3(a), the four absolute maxima of the charge distribution $\rho_{a}$ coincide with the centers of pentagons, ${ }^{12}$ which is very reasonable. Indeed, it is known that in the neutral molecule, the centers of pentagons are the electron-poor regions and any additional negative charge experiences minimal repulsion at these centers. A direct consequence of this fact is the $\operatorname{Pa} \overline{3}$ phase of solid $\mathrm{C}_{60}$ : here, an 
a

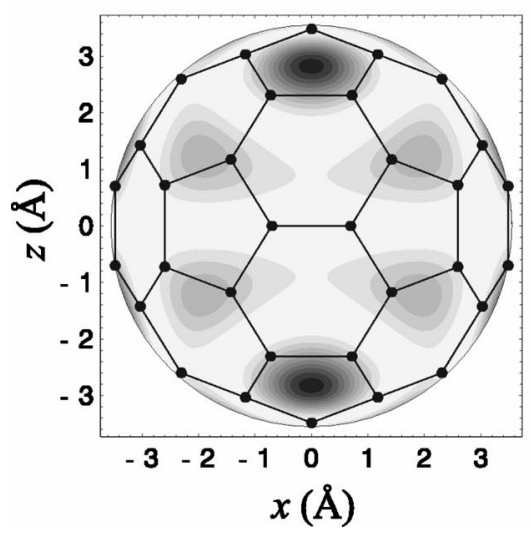

b

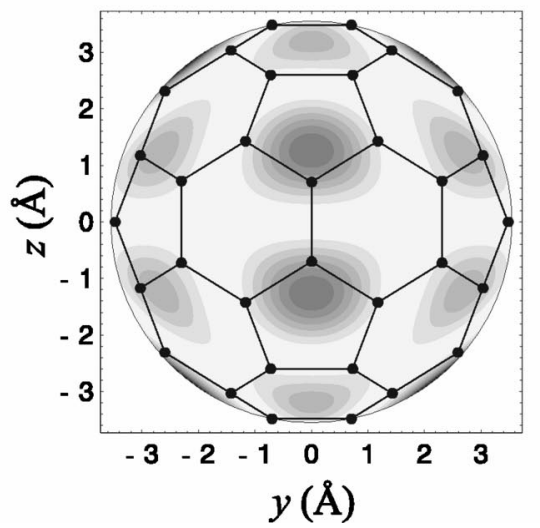

$\rho_{a}$

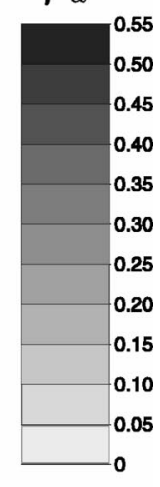

FIG. 3. Angular part $\rho_{a}(\theta, \phi)$ of the charge distribution of $\mathrm{C}_{60}{ }^{-}$monomer in the standard orientation, plotted as a contourplot on a sphere with radius $R=3.55 \AA$, together with the $\mathrm{C}_{60}$ cage. (a) Projection of $\rho_{a}$ onto the $(\vec{a}, \vec{c})$ plane. (b) Projection of $\rho_{a}$ onto the $(\vec{b}, \vec{c})$ plane. The coordinates $(x, y, z)$ and $(\theta, \phi)$ are related to each other via Eq. (2.7) and the constraint $\sqrt{x^{2}+y^{2}+z^{2}}=R$. On both projections, one can see clearly that the charge is mainly concentrated in the equatorial region $(x=0)$. Note the local maxima in the charge density near the $\mathrm{C}$ atoms participating in the cycloaddition bonds, and the four absolute maxima located at the centers of pentagons (electron-poor regions of the neutral $\mathrm{C}_{60}$ molecule).

electron-rich region of one molecule (double $\mathrm{C}-\mathrm{C}$ bond) faces a pentagon of its neighbor. ${ }^{21}$

\section{POTENTIAL ENERGY CALCULATIONS}

The potential energy $U$ of two interacting $\mathrm{C}_{60}^{-}$monomers can now be written down explicitly. Combining Eqs. (3.3a), (3.3b), (4.1a), and (4.1b), one gets

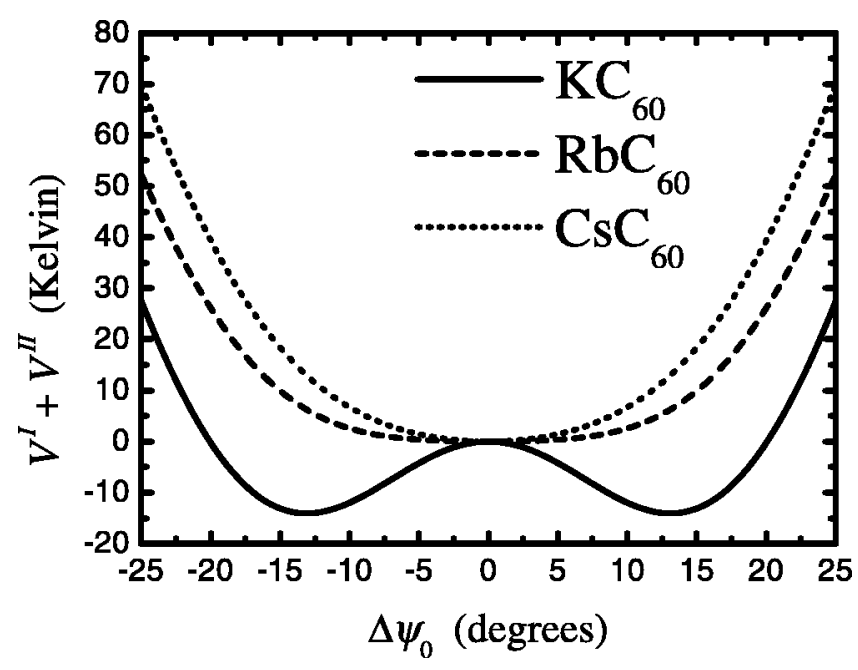

FIG. 4. Dependence of the potential energy $V^{\mathrm{I}}+V^{\mathrm{II}}$ (units of kelvin), to which the total potential energy of the crystal due to $\mathrm{C}_{60}^{-}-\mathrm{C}_{60}^{-}$interactions is proportional, on the deviation angle $\Delta \psi_{0}$ (units of degrees). The energy scale has been shifted for all curves so that the undistorted structure $\left(\Delta \psi_{0}=0^{\circ}\right)$ corresponds to zero potential energy. The double minimum for $\mathrm{KC}_{60}$ (solid line) shows that an energetically more favorable configuration than the Pmnn structure exists, featuring rotated electronic densities, while for $\mathrm{RbC}_{60}$ (dashed line) and $\mathrm{CsC}_{60}$ (dotted line) deviations of the electronic distributions on the $\mathrm{C}_{60}^{-}$monomers from the original $\mathrm{I} / \mathrm{m}$ structure do not lower the potential energy.

$$
\begin{aligned}
& U\left(\vec{n}, \vec{n}^{\prime}\right) \\
& =F \int_{0}^{\pi} \sin \theta d \theta \int_{0}^{2 \pi} d \phi \int_{0}^{\pi} \sin \theta^{\prime} d \theta^{\prime} \int_{0}^{2 \pi} d \phi^{\prime} \\
& \quad \times \frac{\rho_{a}(\theta, \phi-\psi(\vec{n})-\Delta \psi(\vec{n})) \rho_{a}\left(\theta^{\prime}, \phi^{\prime}-\psi\left(\vec{n}^{\prime}\right)-\Delta \psi\left(\vec{n}^{\prime}\right)\right)}{D\left(\theta, \phi, \theta^{\prime}, \phi^{\prime} ; \vec{n}, \vec{n}^{\prime}\right)},
\end{aligned}
$$

with

$$
F=\frac{e^{2}}{4 \pi \epsilon_{0}}=167100 \mathrm{~K} \AA
$$

$$
\begin{aligned}
D(\theta, \phi, & \left.\theta^{\prime}, \phi^{\prime} ; \vec{n}, \vec{n}^{\prime}\right) \\
=\{[ & \left.R \cos \theta-R \cos \theta^{\prime}-\left(n_{1}^{\prime}-n_{1}\right) a\right]^{2} \\
& +\left[R \sin \theta \cos \phi-R \sin \theta^{\prime} \cos \phi^{\prime}-\left(n_{2}^{\prime}-n_{2}\right) b\right]^{2} \\
& \left.+\left[R \sin \theta \sin \phi-R \sin \theta^{\prime} \sin \phi^{\prime}-\left(n_{3}^{\prime}-n_{3}\right) c\right]^{2}\right\}^{1 / 2} .
\end{aligned}
$$

The dependence on $\Delta \psi_{0}$ of the quantity $V^{\mathrm{I}}+V^{\mathrm{II}}$, to which $\mathcal{U}$ is proportional, is shown in Fig. 4 for both $\mathrm{KC}_{60}(P m n n$ structure) and $\mathrm{RbC}_{60}(I 2 / m$ structure). The lattice constants used in the calculations are given in Table I. The potential energy for $\mathrm{KC}_{60}$ exhibits a double minimum, which implies that a deviation of the charge distributions from the Pmnn structure as described by the doubling mechanism of Sec. II [Fig. 2(a)] is favored. The optimal configuration corresponds to a deviation angle of $\Delta \psi_{0} \approx 13^{\circ}$. On the other hand, for $\mathrm{RbC}_{60}$, such a deviation [Fig. 2(b)] would never lead to an energetically more favorable structure since the energy minimum lies at $\Delta \psi_{0}=0^{\circ}$. 
TABLE I. Lattice constants of $\mathrm{KC}_{60}$ and $\mathrm{RbC}_{60}$, taken from Ref. 7 ( $T=6 \mathrm{~K}$ values for $\mathrm{KC}_{60}$ and $T=5 \mathrm{~K}$ values for $\mathrm{RbC}_{60}$ ), and of $\mathrm{CsC}_{60}$, taken from Ref. 13 ( $T=20 \mathrm{~K}$ values); units $\AA$.

\begin{tabular}{lccc}
\hline \hline & $a$ & $b$ & $c$ \\
\hline $\mathrm{KC}_{60}$ & 9.1185 & 9.9010 & 14.3467 \\
$\mathrm{RbC}_{60}$ & 9.0887 & 10.0843 & 14.1583 \\
$\mathrm{CsC}_{60}$ & 9.0968 & 10.1895 & 14.1351 \\
\hline \hline
\end{tabular}

For completeness, the result for $\mathrm{CsC}_{60}$ is also shown in Fig. 4. Since the $I 2 / m$ space group angle is the same for both $\mathrm{RbC}_{60}$ and $\mathrm{CsC}_{60}, \psi_{\mathrm{RbC}_{60}}=\psi_{\mathrm{CsC}_{60}}=46^{\circ}, 7,13$ the only difference between $\mathrm{RbC}_{60}$ and $\mathrm{CsC}_{60}$ arises from the different lattice constants (Table I). Intuitively, one would therefore expect no qualitative difference between $\mathrm{RbC}_{60}$ and $\mathrm{CsC}_{60}$. Indeed, Fig. 4 confirms the similarity between $\mathrm{RbC}_{60}$ and $\mathrm{CsC}_{60}$. Hence our model implies the absence of a $(\vec{a}$ $+\vec{c}, \vec{b}, \vec{a}-\vec{c}$ ) superstructure (and a concomitant metalinsulator transition) for $\mathrm{CsC}_{60}$.

At this point, we note that the essential dependence of the potential energy $\mathcal{U}$ due to $\mathrm{C}_{60}{ }^{-}-\mathrm{C}_{60}{ }^{-}$interactions on the distortion angle $\Delta \psi_{0}$ is introduced in Eq. (2.6). There, the deviation angle $\psi(\vec{n})$ at lattice site $\vec{n}$ is defined in such a way that the experimentally found doubling scheme is automatically recovered. However, by assigning to each lattice site $\vec{n}$ an order parameter $S(\vec{n})= \pm 1$, corresponding to a deviation angle $\Delta \psi(\vec{n})= \pm \Delta \psi_{0}$, one can show rigorously that this doubling scheme has the lowest potential energy. This analysis is carried out in detail in Appendix A. There it is shown that the average order parameter $\langle S(\vec{n})\rangle$ is given by

$$
\langle S(\vec{n})\rangle=\eta(-1)^{n_{1}+n_{3},}
$$

where $\eta$ is the order parameter amplitude. The condensation scheme (5.2) is indeed equivalent to Eq. (2.6), expressing periodicity doubling along $\vec{a}$ and $\vec{c}$ and no change in periodicity along $\vec{b}$.

\section{DISPLACEMENTS OF THE ALKALI-METAL IONS}

It is clear from Fig. 4 that the valence electronic density deviations $\Delta \psi(\vec{n})$ from the Pmnn structure are already sufficient to account for the periodicity doubling along the $\vec{a}$ and $\vec{c}$ axes in $\mathrm{KC}_{60}$ observed by the x-ray scattering measurements described in Ref. 11. In this section we investigate the role of the alkali-metal ions.

The ability of the charge distributions on the $\mathrm{C}_{60}{ }^{-}$monomers to rotate creates a picture reminiscent of rotating molecules in molecular crystals. It is well known that orientational motion of molecular ions in molecular crystals influences the translational movements of neighboring counterions. Here, we have an analogous situation and in $\mathrm{KC}_{60}$ one expects average center-of-mass displacements of the $\mathrm{K}^{+}$ ions induced by the angular deviations of the charge on the $\mathrm{C}_{60}{ }^{-}$ions.

The theory of bilinear translation-rotation (TR) coupling a

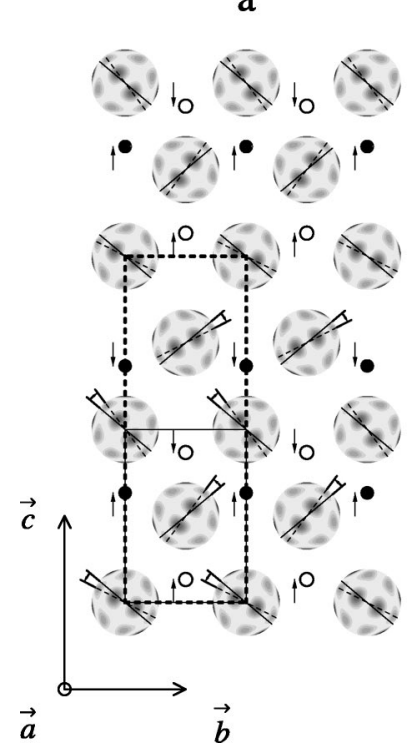

b

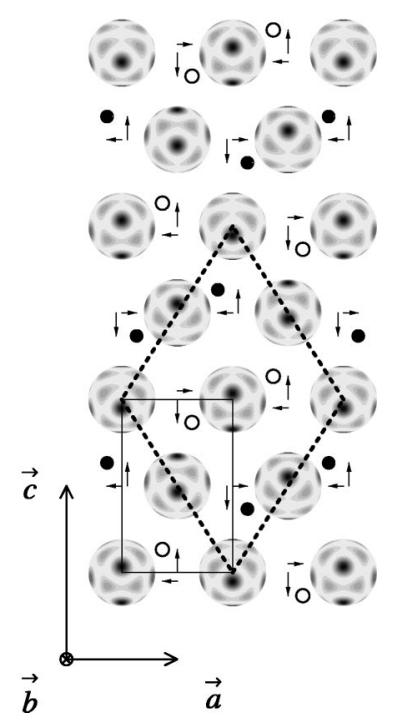

FIG. 5. The full doubling mechanism in $\mathrm{KC}_{60}$ : the charge distributions are rotated $\pm 13^{\circ}$ away from their original Pmnn structure, and the equilibrium positions of the alkali-metal ions are shifted, resulting in a $(\vec{a}+\vec{c}, \vec{b}, \vec{a}-\vec{c})$ superstructure. The charge distributions are represented by their contourplot projections of Fig. 3. The radius of the $\mathrm{C}_{60}{ }^{-}$units has been reduced for clarity. The alkali-metal ions are represented by filled and empty circles. (a) Projection onto the crystallographic $(\vec{b}, \vec{c})$ plane (compare with Fig. 2). (b) Projection onto the crystallographic $(\vec{a}, \vec{c})$ plane.

in molecular crystals ${ }^{22,23}$ is the tool to examine the effect of molecular orientations on lattice displacements of counterions and can be applied here as well. The interactions to be considered are the Coulomb attractions between the $\mathrm{C}_{60}{ }^{-}$ monomers and the $\mathrm{K}^{+}$ions. Full details are given in Appen$\operatorname{dix}$ B. The main result, Eq. (B15), is that average displacements $\left\langle\vec{u}_{\mathrm{A}}\left(\vec{n}_{\mathrm{A}}\right)\right\rangle$ of the alkali-metal ions, labeled by lattice indices $\vec{n}_{\mathrm{A}}=\left(n_{\mathrm{A} 1}, n_{\mathrm{A} 2}, n_{\mathrm{A} 3}\right)$, are found to occur:

$$
\left\langle\vec{u}_{\mathrm{A}}\left(\vec{n}_{\mathrm{A}}\right)\right\rangle=\left(\begin{array}{c}
u_{1} \\
0 \\
u_{3}
\end{array}\right)(-1)^{k_{\mathrm{A}}} .
$$

Here $k_{\mathrm{A}}$ is the integer part of $n_{\mathrm{A} 1}+n_{\mathrm{A} 3}$ :

$$
n_{\mathrm{A} 1}+n_{\mathrm{A} 3}=k_{\mathrm{A}}+\frac{1}{2} \text {. }
$$

Equation (6.1) shows that there are no displacements of the $\mathrm{K}^{+}$ions (and therefore no periodicity doubling) along the $\vec{b}$ axis, and that along the $\vec{a}$ and $\vec{c}$ axes displacements of the $\mathrm{K}^{+}$ions happen in such a way that the periodicity doubling scheme of the charge distributions on the $\mathrm{C}_{60}{ }^{-}$monomers is respected. The average $\mathrm{K}^{+}$displacements - a result of the rotational deviations of the charge distributions-constitute therefore a secondary doubling mechanism and form a part of the structural change in polymerized $\mathrm{KC}_{60}$. As mentioned in the Introduction, displacements of the $\mathrm{K}^{+}$ions were suggested by Coulon et al. ${ }^{11}$ to explain (partly) the structural 
phase transition. The total doubling mechanism, now including the $\mathrm{K}^{+}$displacements, is visualized in Fig. 5.

In $\mathrm{RbC}_{60}$ and $\mathrm{CsC}_{60}$, the charge distributions of the $\mathrm{C}_{60}{ }^{-}$ monomers do not rotate away from the original $I 2 / \mathrm{m}$ structure. The alkali-metal ions will therefore exhibit no average displacements, keeping the $I 2 / m$ structure intact.

\section{DISCUSSION AND CONCLUSIONS}

In the preceding sections, we have suggested and examined the possibility of having small rotations of the valence charge distributions on all $\mathrm{C}_{60}{ }^{-}$monomers in the $A \mathrm{C}_{60}$ alkali-metal fullerides. $\mathrm{In}_{\mathrm{KC}_{60}}$, these electronic density distortions lower the potential energy and result in average center-of-mass displacements of the $\mathrm{K}^{+}$ions. Both the orientational deviations of the charge distributions and the translations of the alkali-metals occur in such a way that the "new" structure has a doubled periodicity along the $\vec{a}$ and $\vec{c}$ directions in comparison with the "old" structure, while the periodicity along the $\vec{b}$ direction does not change. These two structural changes can therefore account for the experimentally observed $(\vec{a}+\vec{c}, \vec{b}, \vec{a}-\vec{c})$ superstructure in $\mathrm{KC}_{60}$. In $\mathrm{RbC}_{60}$, the potential energy is not lowered by deviations of the orientations of the charge densities. Hence, the $I 2 / \mathrm{m}$ structure will be preserved. (Since the charge densities do not deviate from their $I 2 / \mathrm{m}$ equilibrium orientations, there is no driving force to displace the $\mathrm{Rb}^{+}$ions, which will therefore remain at their equilibrium positions.) To summarize, the model we present forms a possible mechanism to explain both the experimentally observed periodicity doubling in $\mathrm{KC}_{60}$ and the absence of a similar doubling scheme in $\mathrm{RbC}_{60}$. It establishes a theoretical basis of "an appealing hypothesis" discussed in Ref. 11, where a combination of a charge density wave (CDW) with large correlated K displacements was suggested as a mechanism for the superstructure.

We recall that our model allows rotations of the charge distributions associated with the $\mathrm{C}_{60}{ }^{-}$monomers in the lattice, while the $\mathrm{C}$ cores remain at fixed positions, i.e., the structure formed by the $\mathrm{C}$ cores does not deviate from the original Pmnn or $I 2 / m$ structure. This immediately launches the question why the $\mathrm{C}$ core network and the electronic distribution around it would behave so "independently" (in $\mathrm{KC}_{60}$ ). First, we note that the angular deviation is relatively small: the energy minimum occurs at $\Delta \psi_{0} \approx 13^{\circ}$. Furthermore, one can argue that any deviation angle $\Delta \psi_{0}$ different from zero already causes an energy lowering, and that it may even be so that the cores of the $\mathrm{C}_{60}$ clusters do "follow" the electronic density distortions, thereby causing some restoring forces that prevent the structure from going as far as the $\Delta \psi_{0} \approx 13^{\circ}$ configuration but rather causing an equilibrium situation at a smaller deviation angle. A smaller equilibrium angle can also be the result of restoring forces that act against the change in chemical bonding between $\mathrm{C}_{60}{ }^{-}$monomers, since the chemical bonding is affected by rotations of the electron distributions on the $\mathrm{C}_{60}{ }^{-}$monomers.

Concerning the accompanying metal-insulator transition in $\mathrm{KC}_{60},{ }^{11}$ we note that the periodicity doubling along the $\vec{a}$ axis via the mechanism described in Sec. II will affect the electron transport properties along the polymer chains. We point out that the suggested doubling scheme along the polymerization axis can be seen as a CDW, however, not in the usual sense of a charge quantity modulation, but a modulation of the orientation of the charge distribution along the polymer chain, which we call an orientational charge density wave (OCDW). The charge of every $\mathrm{C}_{60}$ unit remains the same. It is well known that a one-dimensional electron gas, coupled to the underlying crystal lattice through electronphonon interactions, is unstable. The Peierls instability leads to a CDW accompanied by periodic lattice distortions. ${ }^{24}$ In the case of a complete charge transfer of one electron to each anion, a half-filled band leads to an instability with wave vector $q=2 k_{F}=2(\pi / 2 a)$, which corresponds to a doubling of the unit cell from lattice constant $a$ to $2 a$ in real space. The insulating state results from the opening of an energy gap that separates the filled lower electron band from the empty conduction band. In the present case of an OCDW, the modulation of the orientations of the charge distributions along the polymer chain and the concomitant displacements of the $\mathrm{K}^{+}$ions play the role of the lattice distortions, and the metal-insulator transition is a consequence of the structural transformation. It is not necessarily accompanied by displacements of the $\mathrm{C}_{60}{ }^{-}$monomers along the polymerization direction. Another consequence of the rotations of the electron densities on the $\mathrm{C}_{60}$ units is a decrease of the transfer integrals between neighboring molecules in a polymer chain, which also results in a reduction of the conductivity during the phase transition.

Theoretical work $^{9,10}$ on the unpolymerized $\rightarrow$ polymerized phase transition in the $A \mathrm{C}_{60}, A=\mathrm{K}, \mathrm{Rb}, \mathrm{Cs}$, compounds has revealed that the structural difference of the polymer phases (space group Pmnn for $\mathrm{KC}_{60}, I 2 / m$ for $\mathrm{RbC}_{60}$ and $\mathrm{CsC}_{60}$ ) is due to the electronic quadrupolarizability of the alkali-metals, and not due to some other alkali-metal-specific parameters such as lattice constants or interaction strengths. In our view, the alkali-atom-specificity of the structural phase transition (which is present for $\mathrm{KC}_{60}$ but absent for $\mathrm{RbC}_{60}$ ) studied here is again not due to lattice constants, but is a direct consequence of the different space groups of the two compounds studied. One can therefore say that it is again the electronic quadrupolarizability causingindirectly-the structural difference of $\mathrm{KC}_{60}$ and $\mathrm{RbC}_{60}$, since it is responsible for the different space groups.

In conclusion, we have presented a model that (i) explains the occurrence of a second structural phase transition in $\mathrm{KC}_{60}$ and the absence of such a transition in $\mathrm{RbC}_{60}$, both observed experimentally, and (ii) is a starting point for investigations concerning the electronic properties of the alkalimetal fullerides in general and the experimentally observed metal-insulator transition in $\mathrm{KC}_{60}$ in particular.

\section{ACKNOWLEDGMENTS}

We acknowledge useful discussions with P. Launois, R. Moret, and A. Pénicaud. This work has been financially supported by the Fonds voor Wetenschappelijk Onderzoek, 
Vlaanderen, and by the Bijzonder Onderzoeksfonds, Universiteit Antwerpen, UIA.

\section{APPENDIX A: CONDENSATION SCHEME FOR THE STRUCTURAL PHASE TRANSITION}

From Fig. 4, it follows that in $\mathrm{KC}_{60}$, simultaneous deviations of the charge distributions on all $\mathrm{C}_{60}{ }^{-}$monomers, described by the doubling mechanism of Sec. II, lead to an energetically more favorable structure. In this appendix, we show rigorously that this scheme will indeed occur, without making the a priori assumption of alternating electronic deviations along the $\vec{a}$ and $\vec{c}$ axes and equal deviations along the $\vec{b}$ axis, expressed mathematically by Eq. (2.6).

To each site $\vec{n}$, we assign a quantity $S(\vec{n})$, which takes on the values +1 and -1 , corresponding, respectively, to a deviation angle of the electronic density $\Delta \psi(\vec{n})=+\Delta \psi_{0}$ and $-\Delta \psi_{0}$. Allowing only the two deviation angles resulting in minima in the potential energy curve (Fig. 4), $\left|\Delta \psi_{0}\right|$ $=13.0208^{\circ}$, the interaction energy $U(\vec{n}, \vec{n}+\vec{\mu})[$ Eq. (3.2a)] can be written as

$$
\begin{aligned}
U(\vec{n}, \vec{n}+\vec{\mu})= & \frac{1+S(\vec{n})}{2} U^{++}(\vec{n}, \vec{n}+\vec{\mu}) \frac{1+S(\vec{n}+\vec{\mu})}{2} \\
& +\frac{1-S(\vec{n})}{2} U^{-+}(\vec{n}, \vec{n}+\vec{\mu}) \frac{1+S(\vec{n}+\vec{\mu})}{2} \\
& +\frac{1+S(\vec{n})}{2} U^{+-}(\vec{n}, \vec{n}+\vec{\mu}) \frac{1-S(\vec{n}+\vec{\mu})}{2} \\
& +\frac{1-S(\vec{n})}{2} U^{--}(\vec{n}, \vec{n}+\vec{\mu}) \frac{1-S(\vec{n}+\vec{\mu})}{2}
\end{aligned}
$$

where $U^{ \pm+}(\vec{n}, \vec{n}+\vec{\mu})$ is the value of $U(\vec{n}, \vec{n}+\vec{\mu})$ when $S(\vec{n})= \pm 1$ and $S(\vec{n}+\vec{\mu})=1$. Analogously, $U^{ \pm-}(\vec{n}, \vec{n}+\vec{\mu})$ is the value of $U(\vec{n}, \vec{n}+\vec{\mu})$ when $S(\vec{n})= \pm 1$ and $S(\vec{n}+\vec{\mu})$ $=-1$. As in Sec. III, we consider 14 nearest neighbors. The energies $U^{ \pm+}(\vec{n}, \vec{n}+\vec{\mu})$ and $U^{ \pm-}(\vec{n}, \vec{n}+\vec{\mu})$ have been calculated using Eqs. (5.1a)-(5.1c) and are listed in Table II. Since the corner points $\left(\vec{n} \in \mathbb{Z}^{3}\right)$ and the center point $[\vec{n}$ $\left.\in\left(\mathbb{Z}+\frac{1}{2}\right)^{3}\right]$ of the orthorhombic cells have a different chain angle $\left(+\psi_{\mathrm{KC}_{60}}\right.$ and $-\psi_{\mathrm{KC}_{60}}$, respectively; see Eq. (2.2) and Fig. 1), they have to be considered separately.

The total energy $\mathcal{U}$ [Eq. (3.1)] is then obtained by summing $U(\vec{n}, \vec{n}+\vec{\mu})$ over the whole lattice. It is convenient to write the result in Fourier space. Defining the discrete Fourier transform of $S(\vec{n})$ by

$$
\begin{gathered}
S(\vec{n})=\frac{1}{\sqrt{\mathcal{N}}} \sum_{\vec{q}} e^{i \vec{q} \cdot \vec{X}(\vec{n})} S(\vec{q}), \\
S(\vec{q})=\frac{1}{\sqrt{\mathcal{N}}} \sum_{\vec{n}} e^{-i \vec{q} \cdot \vec{X}(\vec{n})} S(\vec{n}),
\end{gathered}
$$

we obtain

$$
\mathcal{U}=\frac{1}{2} \sum_{\vec{n}} \sum_{\vec{\mu}} U(\vec{n}, \vec{n}+\vec{\mu})=\frac{1}{2} \sum_{\vec{q}} J(\vec{q}) S(\vec{q}) S(-\vec{q})+C
$$

Here we have split the summation over $\vec{n}$ into two parts: corner points $\vec{n} \in \mathbb{Z}^{3}$ and center points $\vec{n} \in\left(\mathbb{Z}+\frac{1}{2}\right)^{3}$. The summation over $\vec{n}$ in Eq. (A2b) is understood to be a summation over corner points - of which there are $\mathcal{N}=N / 2$ - only. In Eq. (A3), $C$ is an irrelevant constant and

$$
\begin{gathered}
J(\vec{q})=J_{a} \cos \left(q_{X} a\right)+J_{b} \cos \left(q_{Y} b\right)+J_{c} \cos \left(q_{Z} c\right) \\
+J_{a b c} \cos \left(q_{X} \frac{a}{2}\right) \cos \left(q_{Y} \frac{b}{2}\right) \cos \left(q_{Z} \frac{c}{2}\right) \\
J_{a}=2\left(J_{a}^{+}-J_{a}^{-}\right), \\
J_{b}=J_{b}^{++}-2 J_{b}^{-}+J_{b}^{--}, \\
J_{c}=J_{c}^{++}-2 J_{c}^{-}+J_{c}^{--}, \\
J_{a b c}=4\left(2 J_{a b c}^{+}-J_{a b c}^{-+}-J_{a b c}^{+-}\right) .
\end{gathered}
$$

The coefficients $J_{a}^{+}, \ldots, J_{a b c}^{+-}$are related to the potential energies $U^{++}, \ldots, U^{--}$as is indicated in Table II. We now determine the absolute minimum of the function $J(\vec{q})$ in reciprocal space. Taking into account the numerical values of $J_{a}=6 \mathrm{~K}, J_{b}=-14 \mathrm{~K}, J_{c}=1 \mathrm{~K}$, and $J_{a b c}=12 \mathrm{~K}$, one finds that the absolute minimum lies at $\vec{q}=\left(q_{X}, q_{Y}, q_{Z}\right)$ $=(\pi / a, 0, \pi / c) \equiv \vec{q}_{B}$. The dominance of $J\left(\vec{q}_{B}\right)$ leads to a condensation of $S(\vec{q})$ at $\vec{q}=\vec{q}_{B}$ :

$$
\langle S(\vec{q})\rangle=\eta \sqrt{\mathcal{N}} \delta_{q}, \vec{q}_{B}
$$

Here $\eta$ is the order parameter amplitude. The condensation scheme in Fourier space (A5) corresponds to the following real space condensation scheme:

$$
\langle S(\vec{n})\rangle=\eta \cos \left[\vec{q}_{B} \cdot \vec{X}(\vec{n})\right]=\eta(-1)^{n_{1}+n_{3}}
$$

\section{APPENDIX B:}

\section{TRANSLATION-ROTATION COUPLING IN $\mathrm{KC}_{60}$}

In this appendix, we examine the coupling between the orientational deviations of the charge distributions on the $\mathrm{C}_{60}{ }^{-}$monomers-lowering the crystal's potential energy in $\mathrm{KC}_{60}$ (see Fig. 4) —and displacements of the $\mathrm{K}^{+}$ions. We use concepts of the theory of bilinear translation-rotation 
TABLE II. Energies $U^{++}, U^{-+}, U^{+-}$and $U^{--}$, calculated for the 14 nearest neighbors whose relative lattice indices $\vec{\mu}$ are listed in the first column. A distinction between the corner points (upper part of the table) and the center point (lower part of the table) of the orthorhombic cells has to be made. Because of symmetry reasons, only a limited number of different numerical values occurs.

\begin{tabular}{|c|c|c|c|c|}
\hline$\vec{\mu}$ & $U^{++}(\vec{n}, \vec{n}+\vec{\mu})$ & $\begin{array}{c}\vec{n} \in \mathbb{Z}^{3} \\
U^{-+}(\vec{n}, \vec{n}+\vec{\mu})\end{array}$ & $U^{+-}(\vec{n}, \vec{n}+\vec{\mu})$ & $U^{--}(\vec{n}, \vec{n}+\vec{\mu})$ \\
\hline$(1,0,0)$ & $J_{a}^{+}=18203 \mathrm{~K}$ & $J_{a}^{-}=18200 \mathrm{~K}$ & $J_{a}^{-}$ & $J_{a}^{+}$ \\
\hline$(0,1,0)$ & $J_{b}^{++}=16946 \mathrm{~K}$ & $J_{b}^{-}=17060 \mathrm{~K}$ & $J_{b}^{-}$ & $J_{b}^{--}=17160 \mathrm{~K}$ \\
\hline$(-1,0,0)$ & $J_{a}^{+}$ & $J_{a}^{-}$ & $J_{a}^{-}$ & $J_{a}^{+}$ \\
\hline$(0,-1,0)$ & $J_{b}^{++}$ & $J_{b}^{-}$ & $J_{b}^{-}$ & $J_{b}^{--}$ \\
\hline$(0,0,1)$ & $J_{c}^{++}=11708 \mathrm{~K}$ & $J_{c}^{-}=11669 \mathrm{~K}$ & $J_{c}^{-}$ & $J_{c}^{--}=11631 \mathrm{~K}$ \\
\hline$(0,0,-1)$ & $J_{c}^{++}$ & $J_{c}^{-}$ & $J_{c}^{-}$ & $J_{c}^{--}$ \\
\hline$\left(\frac{1}{2}, \frac{1}{2}, \frac{1}{2}\right)$ & $J_{a b c}^{+}=16980 \mathrm{~K}$ & $J_{a b c}^{-+}=16954 \mathrm{~K}$ & $J_{a b c}^{+-}=17003 \mathrm{~K}$ & $J_{a b c}^{+}$ \\
\hline$\left(-\frac{1}{2}, \frac{1}{2}, \frac{1}{2}\right)$ & $J_{a b c}^{+}$ & $J_{a b c}^{-+}$ & $J_{a b c}^{+-}$ & $J_{a b c}^{+}$ \\
\hline$\left(-\frac{1}{2},-\frac{1}{2}, \frac{1}{2}\right)$ & $J_{a b c}^{+}$ & $J_{a b c}^{-+}$ & $J_{a b c}^{+-}$ & $J_{a b c}^{+}$ \\
\hline$\left(\frac{1}{2},-\frac{1}{2}, \frac{1}{2}\right)$ & $J_{a b c}^{+}$ & $J_{a b c}^{-+}$ & $J_{a b c}^{+-}$ & $J_{a b c}^{+}$ \\
\hline$\left(\frac{1}{2}, \frac{1}{2},-\frac{1}{2}\right)$ & $J_{a b c}^{+}$ & $J_{a b c}^{-+}$ & $J_{a b c}^{+-}$ & $J_{a b c}^{+}$ \\
\hline$\left(-\frac{1}{2}, \frac{1}{2},-\frac{1}{2}\right)$ & $J_{a b c}^{+}$ & $J_{a b c}^{-+}$ & $J_{a b c}^{+-}$ & $J_{a b c}^{+}$ \\
\hline$\left(-\frac{1}{2},-\frac{1}{2},-\frac{1}{2}\right)$ & $J_{a b c}^{+}$ & $J_{a b c}^{-+}$ & $J_{a b c}^{+-}$ & $J_{a b c}^{+}$ \\
\hline$\left(\begin{array}{ccc}1 & 1 & 1 \\
- & --\end{array}\right)$ & $J_{a b c}^{+}$ & $J_{a b c}^{-+}$ & $J_{a b c}^{+-}$ & $J_{a b c}^{+}$ \\
\hline $\begin{array}{lll}-1 & 2 & 2\end{array}$ & & $\vec{n} \in\left(\mathrm{Z}+\frac{1}{2}\right)^{3}$ & & \\
\hline$\vec{\mu}$ & $U^{++}(\vec{n}, \vec{n}+\vec{\mu})$ & $U^{-+}(\vec{n}, \vec{n}+\vec{\mu})$ & $U^{+-}(\vec{n}, \vec{n}+\vec{\mu})$ & $U^{--}(\vec{n}, \vec{n}+\vec{\mu})$ \\
\hline$(1,0,0)$ & $J_{a}^{+}$ & $J_{a}^{-}$ & $J_{a}^{-}$ & $J_{a}^{+}$ \\
\hline$(0,1,0)$ & $J_{b}^{--}$ & $J_{b}^{-}$ & $J_{b}^{-}$ & $J_{b}^{++}$ \\
\hline$(-1,0,0)$ & $J_{a}^{+}$ & $J_{a}^{-}$ & $J_{a}^{-}$ & $J_{a}^{+}$ \\
\hline$(0,-1,0)$ & $J_{b}^{--}$ & $J_{b}^{-}$ & $J_{b}^{-}$ & $J_{b}^{++}$ \\
\hline$(0,0,1)$ & $J_{c}^{--}$ & $J_{c}^{-}$ & $J_{c}^{-}$ & $J_{c}^{++}$ \\
\hline$(0,0,-1)$ & $J_{c}^{--}$ & $J_{c}^{-}$ & $J_{c}^{-}$ & $J_{c}^{++}$ \\
\hline$\left(\begin{array}{ccc}1 & 1 & 1 \\
- & - & -\end{array}\right)$ & $J_{a b c}^{+}$ & $J_{a b c}^{+-}$ & $J_{a b c}^{-+}$ & $J_{a b c}^{+}$ \\
\hline$\left(\begin{array}{ccc}2 & 2 & 2 \\
1 & 1 & 1 \\
-- & 1 & 1\end{array}\right)$ & $J_{a b c}^{+}$ & $J_{a b c}^{+-}$ & $J_{a b c}^{-+}$ & $J_{a b c}^{+}$ \\
\hline 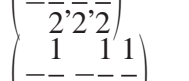 & $J_{a b c}^{+}$ & $J_{a b c}^{+-}$ & $J_{a b c}^{-+}$ & $J_{a b c}^{+}$ \\
\hline 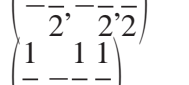 & $J_{a b c}^{+}$ & $J_{a b c}^{+-}$ & $J_{a b c}^{-+}$ & $J_{a b c}^{+}$ \\
\hline 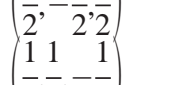 & $J_{a b c}^{+}$ & $J_{a b c}^{+-}$ & $J_{a b c}^{-+}$ & $J_{a b c}^{+}$ \\
\hline$\left(\begin{array}{ccc}\overline{2}, \overline{2} & -\overline{2} \\
1 & 1 & 1 \\
- & 1 & 1\end{array}\right)$ & $J_{a b c}^{+}$ & $J_{a b c}^{+-}$ & $J_{a b c}^{-+}$ & $J_{a b c}^{+}$ \\
\hline$\left(\begin{array}{c}-\overline{2}, \overline{2},-\overline{2} \\
1 \\
-1\end{array}\right.$ & $J_{a b c}^{+}$ & $J_{a b c}^{+-}$ & $J_{a b c}^{-+}$ & $J_{a b c}^{+}$ \\
\hline$\left(\begin{array}{l}-\frac{-}{2},-\frac{-}{2},-\frac{-}{2} \\
\frac{1}{2},-\frac{1}{2},-\frac{1}{2}\end{array}\right)$ & $J_{a b c}^{+}$ & $J_{a b c}^{+-}$ & $J_{a b c}^{-+}$ & $J_{a b c}^{+}$ \\
\hline
\end{tabular}


(TR) coupling in molecular crystals, ${ }^{22,23}$ which is generally used to determine the influence of molecular orientations on lattice displacements of counterions.

The starting point is the potential energy of a $\mathrm{C}_{60}{ }^{-}$monomer and $\mathrm{a} \mathrm{K}^{+}$ion:

$U\left(\vec{n}, \vec{n}_{\mathrm{A}}\right)=\frac{1}{4 \pi \epsilon_{0}} \int d \vec{r} \int d \vec{r}_{\mathrm{A}} \frac{\rho(\vec{r} ; \psi(\vec{n})+\Delta \psi(\vec{n})) \rho_{\mathrm{A}}\left(\vec{r}_{\mathrm{A}}\right)}{\left|\vec{r}-\vec{r}_{\mathrm{A}}-\vec{X}\left(\vec{n}_{\mathrm{A}}-\vec{n}\right)-\vec{u}_{\mathrm{A}}\left(\vec{n}_{\mathrm{A}}\right)\right|}$,

where the subscript ${ }_{A}$ refers to the alkali-metal ion. Similarly as in Eq. (3.2a), the integration variables $\vec{r}$ and $\vec{r}_{\mathrm{A}}$ in Eq. (B1) refer to the local coordinate systems associated with the lattice sites $\vec{n}$ and $\vec{n}_{\mathrm{A}}$, respectively, being the reason for the appearance of the relative lattice vector $\vec{X}\left(\vec{n}_{\mathrm{A}}-\vec{n}\right)$ and the alkali-metal lattice displacement vector $\vec{u}_{\mathrm{A}}\left(\vec{n}_{\mathrm{A}}\right)$. To be consistent with the earlier convention

$$
\left\{\begin{array}{l}
n_{1} \in \mathbb{Z} \\
n_{2} \in \mathbb{Z} \\
n_{3} \in \mathbb{Z}
\end{array}\right\} \text { or }\left\{\begin{array}{l}
n_{1} \in \mathbb{Z}+\frac{1}{2} \\
n_{2} \in \mathbb{Z}+\frac{1}{2} \\
n_{3} \in \mathbb{Z}+\frac{1}{2}
\end{array}\right\},
$$

one must have for the alkali-metal lattice indices $\vec{n}_{\mathrm{A}}$ :

$$
\left\{\begin{array}{l}
n_{\mathrm{A} 1} \in \mathbb{Z} \\
n_{\mathrm{A} 2} \in \mathbb{Z} \\
n_{\mathrm{A} 3} \in \mathbb{Z}+\frac{1}{2}
\end{array}\right\} \text { or }\left\{\begin{array}{l}
n_{\mathrm{A} 1} \in \mathbb{Z}+\frac{1}{2} \\
n_{\mathrm{A} 2} \in \mathbb{Z}+\frac{1}{2} \\
n_{\mathrm{A} 3} \in \mathbb{Z}
\end{array}\right\} \text {. }
$$

We treat the $\mathrm{K}^{+}$ion as a point charge and write for its charge distribution:

$$
\rho_{\mathrm{A}}\left(\vec{r}_{\mathrm{A}}\right)=e \delta\left(\vec{r}_{\mathrm{A}}\right)
$$

Working with the charge distribution $\rho(\vec{r} ; \psi(\vec{n})+\Delta \psi(\vec{n}))$ $=\rho(r, \theta, \phi-\psi(\vec{n})-\Delta \psi(\vec{n}))$ of Sec. IV for the $\mathrm{C}_{60}{ }^{-}$monomer and taking into account Eq. (B4), we get the following expression for $U\left(\vec{n}, \vec{n}_{\mathrm{A}}\right)$ :

$$
U\left(\vec{n}, \vec{n}_{\mathrm{A}}\right)=F \int_{0}^{\pi} \sin \theta d \theta \int_{0}^{2 \pi} d \phi \frac{\rho_{a}(\theta, \phi-\psi(\vec{n})-\Delta \psi(\vec{n}))}{d\left(\theta, \phi ; \vec{n}, \vec{n}_{\mathrm{A}}\right)}
$$

$$
\begin{aligned}
d(\theta, \phi ; & \left.\vec{n}, \vec{n}_{\mathrm{A}}\right) \\
= & \left\{\left[R \cos \theta-\left(n_{\mathrm{A} 1}-n_{1}\right) a-u_{\mathrm{A} 1}\left(\vec{n}_{\mathrm{A}}\right)\right]^{2}\right. \\
& +\left[R \sin \theta \cos \phi-\left(n_{\mathrm{A} 2}-n_{2}\right) b-u_{\mathrm{A} 2}\left(\vec{n}_{\mathrm{A}}\right)\right]^{2} \\
& \left.+\left[R \sin \theta \sin \phi-\left(n_{\mathrm{A} 3}-n_{3}\right) c-u_{\mathrm{A} 3}\left(\vec{n}_{\mathrm{A}}\right)\right]^{2}\right\}^{1 / 2},
\end{aligned}
$$

where the constant $F$ is given by Eq. (5.1b).

We consider small center-of-mass displacements of the $\mathrm{K}^{+}$ions and expand $U\left(\vec{n}, \vec{n}_{\mathrm{A}}\right)$ in terms of the components of $\vec{u}_{\mathrm{A}}\left(\vec{n}_{\mathrm{A}}\right)$, retaining only the zeroth- and first-order terms:

$$
\begin{aligned}
& U\left(\vec{n}, \vec{n}_{\mathrm{A}}\right) \\
& \quad=\left.U\left(\vec{n}, \vec{n}_{\mathrm{A}}\right)\right|_{\vec{u}_{\mathrm{A}}\left(\vec{n}_{\mathrm{A}}\right)=\overrightarrow{0}}+\left.\sum_{i=1}^{3} \frac{\partial U\left(\vec{n}, \vec{n}_{\mathrm{A}}\right)}{\partial u_{\mathrm{A} i}\left(\vec{n}_{\mathrm{A}}\right)}\right|_{\vec{u}_{\mathrm{A}}\left(\vec{n}_{\mathrm{A}}\right)=\overrightarrow{0}} u_{\mathrm{A} i}\left(\vec{n}_{\mathrm{A}}\right) .
\end{aligned}
$$

As in Appendix A, we introduce the quantity $S(\vec{n})= \pm 1$, corresponding to $\Delta \psi(\vec{n})= \pm\left|\Delta \psi_{0}\right|$, with $\left|\Delta \psi_{0}\right|=13.0208^{\circ}$. The interaction energy $U\left(\vec{n}, \vec{n}_{\mathrm{A}}\right)$ can then be written as

$$
U\left(\vec{n}, \vec{n}_{\mathrm{A}}\right)=\frac{1+S(\vec{n})}{2} U^{+}\left(\vec{n}, \vec{n}_{\mathrm{A}}\right)+\frac{1-S(\vec{n})}{2} U^{-}\left(\vec{n}_{,} \vec{n}_{\mathrm{A}}\right)
$$

where $U^{ \pm}\left(\vec{n}, \vec{n}_{\mathrm{A}}\right)$ is the value of $U\left(\vec{n}, \vec{n}_{\mathrm{A}}\right)$ when $S(\vec{n})=$ \pm 1 . The expansion (B6) becomes

$$
\begin{aligned}
U\left(\vec{n}, \vec{n}_{\mathrm{A}}\right)= & \frac{1+S(\vec{n})}{2}\left\{V^{+}\left(\vec{n}, \vec{n}_{\mathrm{A}}\right)+\sum_{i=1}^{3} v_{i}^{+}\left(\vec{n}, \vec{n}_{\mathrm{A}}\right) u_{\mathrm{A} i}\left(\vec{n}_{\mathrm{A}}\right)\right\} \\
& +\frac{1-S(\vec{n})}{2}\left\{V^{-}\left(\vec{n}, \vec{n}_{\mathrm{A}}\right)\right. \\
& \left.+\sum_{i=1}^{3} v_{i}^{-}\left(\vec{n}, \vec{n}_{\mathrm{A}}\right) u_{\mathrm{A} i}\left(\vec{n}_{\mathrm{A}}\right)\right\}
\end{aligned}
$$

with

$$
\begin{gathered}
V^{ \pm}\left(\vec{n}, \vec{n}_{\mathrm{A}}\right)=\left.U^{ \pm}\left(\vec{n}_{,} \vec{n}_{\mathrm{A}}\right)\right|_{\vec{u}_{\mathrm{A}}\left(\vec{n}_{\mathrm{A}}\right)=\overrightarrow{0},} \\
v_{i}^{ \pm}\left(\vec{n}, \vec{n}_{\mathrm{A}}\right)=\left.\frac{\partial U^{ \pm}\left(\vec{n}, \vec{n}_{\mathrm{A}}\right)}{\partial u_{\mathrm{A} i}\left(\vec{n}_{\mathrm{A}}\right)}\right|_{\vec{u}_{\mathrm{A}}\left(\vec{n}_{\mathrm{A}}\right)=\overrightarrow{0}} .
\end{gathered}
$$

The contribution $\mathcal{U}_{\mathrm{C}_{60}-A}$ of all electrostatic $\mathrm{C}_{60}{ }^{-}{ }^{-} \mathrm{K}^{+}$interactions to the potential energy is obtained by summing $U\left(\vec{n}, \vec{n}_{\mathrm{A}}\right)$ over the whole crystal lattice: 


$$
\mathcal{U}_{\mathrm{C}_{60}-\mathrm{A}}=\mathcal{U}^{\mathrm{R}}+\mathcal{U}^{\mathrm{TR}}
$$

where

$$
\begin{gathered}
\mathcal{U}^{\mathrm{R}}=\sum_{\vec{n}} \sum_{\vec{\mu}_{\mathrm{A}}}\left\{\frac{1+S(\vec{n})}{2} V^{+}\left(\vec{n}, \vec{n}+\vec{\mu}_{\mathrm{A}}\right)\right. \\
\left.+\frac{1-S(\vec{n})}{2} V^{-}\left(\vec{n}, \vec{n}+\vec{\mu}_{\mathrm{A}}\right)\right\}, \\
\mathcal{U}^{\mathrm{TR}}=\sum_{\vec{n}} \sum_{\vec{\mu}_{\mathrm{A}}}\left\{\frac{1+S(\vec{n})}{2} \sum_{i=1}^{3} v_{i}^{+}\left(\vec{n}, \vec{n}+\vec{\mu}_{\mathrm{A}}\right) u_{\mathrm{A} i}\left(\vec{n}+\vec{\mu}_{\mathrm{A}}\right)\right. \\
\left.+\frac{1-S(\vec{n})}{2} \sum_{i=1}^{3} v_{i}^{-}\left(\vec{n}, \vec{n}+\vec{\mu}_{\mathrm{A}}\right) u_{\mathrm{A} i}\left(\vec{n}+\vec{\mu}_{\mathrm{A}}\right)\right\} .
\end{gathered}
$$

For a given $\mathrm{C}_{60}{ }^{-}$monomer, we limit ourselves to the six nearest alkali-metal neighbors. The values of $V^{ \pm}\left(\vec{n}, \vec{n}+\vec{\mu}_{\mathrm{A}}\right)$ and $v_{i}^{ \pm}\left(\vec{n}, \vec{n}+\vec{\mu}_{\mathrm{A}}\right), i=1,2,3$ are listed in Table III. As in Appendix A, a distinction has to be made between the corner points $\left(\vec{n} \in \mathbb{Z}^{3}\right)$ and the center point $\left[\vec{n} \in\left(\mathbb{Z}+\frac{1}{2}\right)^{3}\right]$ of the orthorhombic cells.

Introducing the discrete Fourier transforms of the alkalimetal ion displacements,

$$
\begin{aligned}
& \vec{u}_{\mathrm{A}}\left(\overrightarrow{n_{\mathrm{A}}}\right)=\frac{1}{\sqrt{\mathcal{N} m_{\mathrm{A}}}} \sum_{\vec{q}} e^{i \vec{q} \cdot \vec{X}\left(\vec{n}_{\mathrm{A}}\right)} \vec{u}_{\mathrm{A}}(\vec{q}), \\
& \vec{u}_{\mathrm{A}}(\vec{q})=\sqrt{\frac{m_{\mathrm{A}}}{\mathcal{N}} \sum_{n_{\mathrm{A}}}} e^{-i \vec{q} \cdot \vec{X}\left(\vec{n}_{\mathrm{A}}\right)} \vec{u}_{\mathrm{A}}\left(\vec{n}_{\mathrm{A}}\right),
\end{aligned}
$$

where $m_{\mathrm{A}}$ is the mass of the $\mathrm{K}^{+}$ion, and using in addition the discrete Fourier transform of $S(\vec{n})$, defined by Eqs. (A2a) and (A2b), we get for the TR term of the potential energy

$$
\mathcal{U}^{\mathrm{TR}}=\frac{1}{\sqrt{m_{\mathrm{A}}}} \sum_{\vec{q}} S(-\vec{q}) \vec{v}(\vec{q}) \cdot \vec{u}_{\mathrm{A}}(\vec{q})
$$

with

$$
\vec{v}(\vec{q})=i\left(\begin{array}{c}
-2 \mathcal{D}_{1 a b} \sin \left(q_{X} a / 2\right) \cos \left(q_{Y} b / 2\right)\left[1-e^{i\left(q_{X} a / 2+q_{Y} b / 2+q_{Z} c / 2\right)}\right] \\
-2 \mathcal{D}_{2 a b} \cos \left(q_{X} a / 2\right) \sin \left(q_{Y} b / 2\right)\left[1-e^{i\left(q_{X} a / 2+q_{Y} b / 2+q_{Z} c / 2\right)}\right]+\mathcal{D}_{2 c} \sin \left(q_{Z} c / 2\right)\left[1+e^{i\left(q_{X} a / 2+q_{Y} b / 2+q_{Z} c / 2\right)}\right] \\
2 \mathcal{D}_{3 a b} \cos \left(q_{X} a / 2\right) \sin \left(q_{Y} b / 2\right)\left[1+e^{i\left(q_{X} a / 2+q_{Y} b / 2+q_{Z} c / 2\right)}\right]-\mathcal{D}_{3 c} \sin \left(q_{Z} c / 2\right)\left[1-e^{i\left(q_{X} a / 2+q_{Y} b / 2+q_{Z} c / 2\right)}\right]
\end{array}\right)
$$

and

$$
\begin{gathered}
\mathcal{D}_{1 a b}=v_{1 a b}^{+}-v_{1 a b}^{-}, \\
\mathcal{D}_{2 a b}=v_{2 a b}^{+}-v_{2 a b}^{-}, \\
\mathcal{D}_{2 c}=v_{2 c}^{+}-v_{2 c}^{-}, \\
\mathcal{D}_{3 a b}=v_{3 a b}^{+}-v_{3 a b}^{-}, \\
\mathcal{D}_{3 c}=v_{3 c}^{+}-v_{3 c}^{-} .
\end{gathered}
$$

In the theory of bilinear TR coupling in orientationally disordered crystals, ${ }^{23,25}$ it is shown that the minimal potentialenergy for a given orientational configuration $\{S(\vec{q})\}$ is obtained when

$$
\vec{u}_{\mathrm{A}}(\vec{q})=-M^{-1}(\vec{q}) \vec{v}(-\vec{q}) S(\vec{q})
$$

where $M(\vec{q})$ is the dynamical matrix of the orthorhombic crystal in absence of TR coupling. Using for $S(\vec{q})$ the minimal potential energy condensation scheme of Appendix A, Eq. (A5), we find for the displacements in reciprocal space:

$$
\left\langle\vec{u}_{\mathrm{A}}(\vec{q})\right\rangle=-M^{-1}\left(\vec{q}=\vec{q}_{B}\right) i\left(\begin{array}{c}
-4 \mathcal{D}_{1 a b} \\
0 \\
-2 \mathcal{D}_{3 c}
\end{array}\right) \eta \sqrt{\mathcal{N}} \delta_{\vec{q}, \vec{q}_{B}}
$$


TABLE III. Energies $V^{ \pm}$and derivatives of energies $v_{i}^{ \pm}, i=1,2,3$, calculated for the six nearest alkali-metal neighbors with relative lattice indices $\vec{\mu}_{\mathrm{A}}$. A distinction between the corner points (upper part of the table) and the center point (lower part of the table) of the orthorhombic cells has to be made. Only a limited number of different numerical values occurs.

\begin{tabular}{|c|c|c|c|c|}
\hline \multirow[b]{2}{*}{$\vec{\mu}_{\mathrm{A}}$} & \multirow[b]{2}{*}{$V^{+}\left(\vec{n}, \vec{n}+\vec{\mu}_{\mathrm{A}}\right)$} & \multicolumn{2}{|l|}{$\vec{n} \in \mathbb{Z}^{3}$} & \multirow[b]{2}{*}{$v_{3}^{+}\left(\vec{n}, \vec{n}+\vec{\mu}_{\mathrm{A}}\right)$} \\
\hline & & $v_{1}^{+}\left(\vec{n}, \vec{n}+\vec{\mu}_{\mathrm{A}}\right)$ & $v_{2}^{+}\left(\vec{n}, \vec{n}+\vec{\mu}_{\mathrm{A}}\right)$ & \\
\hline$\left(\frac{1}{2}, \frac{1}{2}, 0\right)$ & $V_{a b}^{+}=24665.3 \mathrm{~K}$ & $-v_{1 a b}^{+}=-2481.7 \mathrm{~K} \AA^{-1}$ & $-v_{2 a b}^{+}=-2606.9 \mathrm{~K} \AA^{-1}$ & $+v_{3 a b}^{+}=85.8 \mathrm{~K} \AA^{-1}$ \\
\hline$\left(-\frac{1}{2}, \frac{1}{2}, 0\right)$ & $V_{a b}^{+}$ & $+v_{1 a b}^{+}$ & $-v_{2 a b}^{+}$ & $+v_{3 a b}^{+}$ \\
\hline$\left(-\frac{1}{2},-\frac{1}{2}, 0\right)$ & $V_{a b}^{+}$ & $+v_{1 a b}^{+}$ & $+v_{2 a b}^{+}$ & $-v_{3 a b}^{+}$ \\
\hline$\left(\frac{1}{2},-\frac{1}{2}, 0\right)$ & $V_{a b}^{+}$ & $-v_{1 a b}^{+}$ & $+v_{2 a b}^{+}$ & $-v_{3 a b}^{+}$ \\
\hline$\left(0,0, \frac{1}{2}\right)$ & $V_{c}^{+}=23685.6 \mathrm{~K}$ & $0 \mathrm{~K} \AA^{-1}$ & $+v_{2 c}^{+}=58.3 \mathrm{~K}^{-1}$ & $-v_{3 c}^{+}=-3476.6 \mathrm{~K} \AA^{-1}$ \\
\hline$\left(0,0,-\frac{1}{2}\right)$ & $V_{c}^{+}$ & $0 \mathrm{~K} \AA^{-1}$ & $-v_{2 c}^{+}$ & $+v_{3 c}^{+}$ \\
\hline$\vec{\mu}_{\mathrm{A}}$ & $V^{-}\left(\vec{n}, \vec{n}+\vec{\mu}_{\mathrm{A}}\right)$ & $v_{1}^{-}\left(\vec{n}, \vec{n}+\vec{\mu}_{\mathrm{A}}\right)$ & $v_{2}^{-}\left(\vec{n}, \vec{n}+\vec{\mu}_{\mathrm{A}}\right)$ & $v_{3}^{-}\left(\vec{n}, \vec{n}+\vec{\mu}_{\mathrm{A}}\right)$ \\
\hline$\left(\frac{1}{2}, \frac{1}{2}, 0\right)$ & $V_{a b}^{-}=24821.3 \mathrm{~K}$ & $-v_{1 a b}^{-}=-2531.4 \mathrm{~K}^{-1}$ & $-v_{2 a b}^{-}=-2636.5 \mathrm{~K} \AA^{-1}$ & $+v_{3 a b}^{-}=49.1 \mathrm{~K} \AA^{-1}$ \\
\hline$\left(-\frac{1}{2}, \frac{1}{2}, 0\right)$ & $V_{a b}^{-}$ & $+v_{1 a b}^{-}$ & $-v_{2 a b}^{-}$ & $+v_{3 a b}^{-}$ \\
\hline$\left(-\frac{1}{2},-\frac{1}{2}, 0\right)$ & $V_{a b}^{-}$ & $+v_{1 a b}^{-}$ & $+v_{2 a b}^{-}$ & $-v_{3 a b}^{-}$ \\
\hline$\left(\frac{1}{2},-\frac{1}{2}, 0\right)$ & $V_{a b}^{-}$ & $-v_{1 a b}^{-}$ & $+v_{2 a b}^{-}$ & $-v_{3 a b}^{-}$ \\
\hline$\left(0,0, \frac{1}{2}\right)$ & $V_{c}^{-}=23279.3 \mathrm{~K}$ & $0 \mathrm{~K} \AA^{-1}$ & $+v_{2 c}^{-}=148.3 \mathrm{~K}^{-1}$ & $-v_{3 c}^{-}=-3253.7 \mathrm{~K} \AA^{-1}$ \\
\hline$\left(0,0,-\frac{1}{2}\right)$ & $V_{c}^{-}$ & $0 \mathrm{~K} \AA^{-1}$ & $-v_{2 c}^{-}$ & $+v_{3 c}^{-}$ \\
\hline$\vec{\mu}_{\mathrm{A}}$ & $V^{+}\left(\vec{n}, \vec{n}+\vec{\mu}_{\mathrm{A}}\right)$ & $v_{1}^{+}\left(\vec{n}, \vec{n}+\vec{\mu}_{\mathrm{A}}\right)$ & $v_{2}^{+}\left(\vec{n}, \vec{n}+\vec{\mu}_{\mathrm{A}}\right)$ & $v_{3}^{+}\left(\vec{n}, \vec{n}+\vec{\mu}_{\mathrm{A}}\right)$ \\
\hline$\left(\frac{1}{2}, \frac{1}{2}, 0\right)$ & $V_{a b}^{-}$ & $-v_{1 a b}^{-}$ & $-v_{2 a b}^{-}$ & $-v_{3 a b}^{-}$ \\
\hline$\left(-\frac{1}{2}, \frac{1}{2}, 0\right)$ & $V_{a b}^{-}$ & $+v_{1 a b}^{-}$ & $-v_{2 a b}^{-}$ & $-v_{3 a b}^{-}$ \\
\hline$\left(-\frac{1}{2},-\frac{1}{2}, 0\right)$ & $V_{a b}^{-}$ & $+v_{1 a b}^{-}$ & $+v_{2 a b}^{-}$ & $+v_{3 a b}^{-}$ \\
\hline$\left(\frac{1}{2},-\frac{1}{2}, 0\right)$ & $V_{a b}^{-}$ & $-v_{1 a b}^{-}$ & $+v_{2 a b}^{-}$ & $+v_{3 a b}^{-}$ \\
\hline$\left(0,0, \frac{1}{2}\right)$ & $V_{c}^{-}$ & $0 \mathrm{~K} \AA^{-1}$ & $-v_{2 c}^{-}$ & $-v_{3 c}^{-}$ \\
\hline$\left(0,0,-\frac{1}{2}\right)$ & $V_{c}^{-}$ & $0 \mathrm{~K} \AA^{-1}$ & $+v_{2 c}^{-}$ & $+v_{3 c}^{-}$ \\
\hline
\end{tabular}


TABLE III (Continued).

\begin{tabular}{|c|c|c|c|c|}
\hline$\vec{\mu}_{\mathrm{A}}$ & $V^{-}\left(\vec{n}, \vec{n}+\vec{\mu}_{\mathrm{A}}\right)$ & $\begin{array}{c}\vec{n} \in\left(\mathbb{Z}+\frac{1}{2}\right)^{3} \\
v_{1}^{-}\left(\vec{n}, \vec{n}+\vec{\mu}_{\mathrm{A}}\right)\end{array}$ & $v_{2}^{-}\left(\vec{n}, \vec{n}+\vec{\mu}_{\mathrm{A}}\right)$ & $v_{3}^{-}\left(\vec{n}, \vec{n}+\vec{\mu}_{\mathrm{A}}\right)$ \\
\hline$\left(\frac{1}{2}, \frac{1}{2}, 0\right)$ & $V_{a b}^{+}$ & $-v_{1 a b}^{+}$ & $-v_{2 a b}^{+}$ & $-v_{3 a b}^{+}$ \\
\hline$\left(-\frac{1}{2}, \frac{1}{2}, 0\right)$ & $V_{a b}^{+}$ & $+v_{1 a b}^{+}$ & $-v_{2 a b}^{+}$ & $-v_{3 a b}^{+}$ \\
\hline$\left(-\frac{1}{2},-\frac{1}{2}, 0\right)$ & $V_{a b}^{+}$ & $+v_{1 a b}^{+}$ & $+v_{2 a b}^{+}$ & $+v_{3 a b}^{+}$ \\
\hline$\left(\frac{1}{2},-\frac{1}{2}, 0\right)$ & $V_{a b}^{+}$ & $-v_{1 a b}^{+}$ & $+v_{2 a b}^{+}$ & $+v_{3 a b}^{+}$ \\
\hline$\left(0,0, \frac{1}{2}\right)$ & $V_{c}^{+}$ & $0 \mathrm{~K} \AA^{-1}$ & $-v_{2 c}^{+}$ & $-v_{3 c}^{+}$ \\
\hline$\left(0,0,-\frac{1}{2}\right)$ & $V_{c}^{+}$ & $0 \mathrm{~K}^{-1}$ & $+v_{2 c}^{+}$ & $+v_{3 c}^{+}$ \\
\hline
\end{tabular}

The numerical values of $\mathcal{D}_{1 a b}$ and $\mathcal{D}_{3 c}$ follow from Table III: $\mathcal{D}_{1 a b}=-49.7 \mathrm{~K}^{-1}$ and $\mathcal{D}_{3 c}=222.9 \mathrm{~K}^{-1}$. Since $\vec{q}_{B} \equiv(\pi / a, 0, \pi / c)$, the scheme (B13) becomes in real space

$$
\begin{aligned}
& \left\langle\vec{u}_{\mathrm{A}}\left(\vec{n}_{\mathrm{A}}\right)\right\rangle \\
& =-\frac{1}{\sqrt{m_{\mathrm{A}}}} M^{-1}\left(\vec{q}=\vec{q}_{B}\right)\left(\begin{array}{c}
-4 \mathcal{D}_{1 a b} \\
0 \\
-2 \mathcal{D}_{3 c}
\end{array}\right) \eta i e^{-i \pi\left(n_{\mathrm{A} 1}+n_{\mathrm{A} 3}\right)}
\end{aligned}
$$

The alkali-metal ions are located on a sublattice obeying $n_{\mathrm{A} 1}+n_{\mathrm{A} 3}=k_{\mathrm{A}}+\frac{1}{2}$ with $k_{\mathrm{A}} \in \mathbb{Z}[$ see Eq. (B3)]. Therefore, one has $e^{-i \pi\left(n_{A 1}+n_{A 3}\right)}=-i(-1)^{k_{\mathrm{A}}}$, yielding

$$
\begin{aligned}
\left\langle\vec{u}_{\mathrm{A}}\left(\vec{n}_{\mathrm{A}}\right)\right\rangle & =-\frac{1}{\sqrt{m_{\mathrm{A}}}} M^{-1}\left(\vec{q}=\vec{q}_{B}\right)\left(\begin{array}{c}
-4 \mathcal{D}_{1 a b} \\
0 \\
-2 \mathcal{D}_{3 c}
\end{array}\right) \eta(-1)^{k_{\mathrm{A}}} \\
& =\left(\begin{array}{c}
u_{1} \\
0 \\
u_{3}
\end{array}\right)(-1)^{k_{\mathrm{A}}} .
\end{aligned}
$$

${ }^{1}$ J. Winter and H. Kuzmany, Solid State Commun. 84, 935 (1992); Q. Zhu, O. Zhou, J.E. Fischer, A.R. McGhie, W.J. Romanov, R.M. Strongin, M.A. Cichy, and A.B. Smith III, Phys. Rev. B 47, 13948 (1993).

${ }^{2}$ S. Pekker, L. Forró, L. Mihály, and A. Jánossy, Solid State Commun. 90, 349 (1994).

${ }^{3}$ Q. Zhu, D.E. Cox, and J.E. Fischer, Phys. Rev. B 51, 3966 (1995).

${ }^{4}$ G. Oszlányi, G. Bortel, G. Faigel, M. Tegze, L. Gránásy, S. Pekker, P.W. Stephens, G. Bendele, R. Dinnebier, G. Mihály, A. Jánossy, O. Chauvet, and L. Forró, Phys. Rev. B 51, 12228 (1995).

${ }^{5}$ P.W. Stephens, G. Bortel, G. Faigel, M. Tegze, A. Jánossy, S. Pekker, G. Oszlányi, and L. Forró, Nature (London) 370, 636 (1994).

${ }^{6}$ P. Launois, R. Moret, J. Hone, and A. Zettl, Phys. Rev. Lett. 81, 4420 (1998).

${ }^{7}$ S. Rouzière, S. Margadonna, K. Prassides, and A.N. Fitch, Europhys. Lett. 51, 314 (2000).
${ }^{8}$ L. Forró and L. Mihály, Rep. Prog. Phys. 64, 649 (2001).

${ }^{9}$ K.H. Michel and A.V. Nikolaev, Phys. Rev. Lett. 85, 3197 (2000).

${ }^{10}$ B. Verberck, K.H. Michel, and A.V. Nikolaev, J. Chem. Phys. 116, 10462 (2002).

${ }^{11}$ C. Coulon, A. Pénicaud, R. Clérac, R. Moret, P. Launois, and J. Hone, Phys. Rev. Lett. 86, 4346 (2001).

${ }^{12} \mathrm{P}$. Launois and R. Moret (private communication).

${ }^{13}$ A. Huq, P.W. Stephens, G.M. Bendele, and R.M. Ibberson, Chem. Phys. Lett. 347, 13 (2001).

${ }^{14}$ R.C. Haddon, L.E. Brus, and K. Raghavachari, Chem. Phys. Lett. 125, 459 (1986).

${ }^{15}$ A.V. Nikolaev and K.H. Michel, Solid State Commun. 117, 739 (2001).

${ }^{16}$ K.H. Michel, J.R.D. Copley, and D.A. Neumann, Phys. Rev. Lett. 68, 2929 (1992).

${ }^{17}$ W.I.F. David, R.M. Ibberson, T.J.S. Dennis, J.P. Hare, and K. Prassides, Europhys. Lett. 18, 219 (1992); 18, 735 (addendum) (1992). 
${ }^{18}$ A.V. Nikolaev, K. Prassides, and K.H. Michel, J. Chem. Phys. 108, 4912 (1998).

${ }^{19}$ C. J. Bradley and A. P. Cracknell, The Mathematical Theory of Symmetry in Solids (Clarendon, Oxford, 1972).

${ }^{20}$ T.M. de Swiet, J.L. Yarger, T. Wagberg, J. Hone, B.J. Gross, M. Tomaselli, J.J. Titman, A. Zettl, and M. Mehring, Phys. Rev. Lett. 84, 717 (2000).

${ }^{21}$ W.I.F. David, R.M. Ibberson, J.C. Matthewman, K. Prassides, T.J.S. Dennis, J.P. Hare, H.W. Kroto, R. Taylor, and D.R.M.
Walton, Nature (London) 353, 147 (1991).

${ }^{22}$ K.H. Michel and J. Naudts, Phys. Rev. Lett. 39, 212 (1977); J. Chem. Phys. 67, 547 (1977).

${ }^{23}$ R.M. Lynden-Bell and K.H. Michel, Rev. Mod. Phys. 66, 721 (1994).

${ }^{24}$ R. E. Peierls, Quantum Theory of Solids (Clarendon, Oxford, 1974).

${ }^{25}$ K.H. Michel and J.M. Rowe, Phys. Rev. B 32, 5818 (1985). 